\title{
On $\eta$-Upper Sign Property and Upper Sign Continuity and Their Applications in Equilibrium-Like Problems
}

\author{
Ali Farajzadeh, ${ }^{1}$ Somaye Jafari, ${ }^{1}$ and Chin-Tzong Pang ${ }^{2}$ \\ ${ }^{1}$ Department of Mathematics, Razi University, Kermanshah 67149, Iran \\ ${ }^{2}$ Department of Information Management, Yuan Ze University, Chung-Li 32003, Taiwan \\ Correspondence should be addressed to Chin-Tzong Pang; imctpang@saturn.yzu.edu.tw
}

Received 3 January 2014; Accepted 24 February 2014; Published 27 March 2014

Academic Editor: Chong Li

Copyright (c) 2014 Ali Farajzadeh et al. This is an open access article distributed under the Creative Commons Attribution License, which permits unrestricted use, distribution, and reproduction in any medium, provided the original work is properly cited.

\begin{abstract}
We first introduce the notion of $\eta$-upper sign property which is an extension of the upper sign property introduced in Castellani and Giuli, 2013, by relaxing convexity on the set. Afterwards, we establish a link between the solution sets of local dual equilibrium problem (Minty local equilibrium problem) and equilibrium problem for mappings whose domains are not necessarily convex by relaxing the upper sign continuity on the map, as it is assumed in the literature (Bianchi and Pini, 2005; Castellani and Giuli, 2013; Farajzadeh and Zafarani, 2010). Accordingly, it allows us to extend and obtain some existence results for equilibrium-like problems.
\end{abstract}

\section{Introduction}

Convexity is one of the most significant assumptions in optimization theory and plays an important role in general equilibrium theory. Hanson [1] introduced the concept of invexity as a generalization of convexity for scalar constrained optimization problems. It has been shown by Blum and Oettli [2] and Noor and Oettli [3] that variational inequalities and mathematical programming problems can be viewed as special realization of the abstract equilibrium problems. Equilibrium problems have numerous applications, including but not limited to problems in economics, game theory, finance, traffic analysis, circuit network analysis, and mechanics; see [2-5]. Noor [6] introduced a new class of equilibrium problems called invex equilibrium (or equilibrium-like) problem in the setting of invexity. It has been shown that invex equilibrium problems include variational-like inequality and equilibrium problems as special cases. Hence the invex equilibrium problems cover a vast range of applications. Bianchi and Pini [7] for an equilibrium problem considered its dual equilibrium problem and then presented some results about relationship between the solution set of equilibrium problem and its dual equilibrium by using an extension of the notion of upper sign continuity introduced by Hadjisavvas
[8] (in the setting of variational inequality problems) for bifunctions.

Recently, in the convex setting, Castellani and Giuli [9] introduced the notion of upper sign property. It was shown that, in the framework of variational inequalities, this notion coincides with the upper sign continuity for the setvalued map introduced by Hadjisavvas [8]. However, this phenomenon is not true for bifunctions. In fact, it is not hard to construct examples of bifunctions with upper sign property which is not upper sign continuous and vice versa. Castellani and Giuli [9], by adding a suitable assumption (they called it technical assumption), proved the fact that an upper sign continuous bifunction has upper sign property. In this work, we first extend the concept of upper sign property for bifunctions whose domains are invex. Afterwards, by using two technical assumptions we highlight bifunctions with upper sign property. It is worth noting that we are going to relax the upper sign continuity assumption on the bifunction in order to establish a link between the solution sets of dual equilibrium and equilibrium problems. This result confirms that we can replace the upper sing continuity by some sufficient conditions under which a bifunction has upper sign property. Finally we present some existence results for invex equilibrium problems. The results obtained in 
the paper extend the corresponding results presented in the literature.

\section{Preliminaries}

Let $X$ be a topological vector spaces and $X^{*}$ its topological dual. Let $K$ be a nonempty subset of $X$ and $f: K \times K \rightarrow \mathbb{R}$ (real numbers) and $\eta(\cdot, \cdot): K \times K \rightarrow X$ two functions.

Definition 1. Let $u \in K$. The set $K$ is said to be invex at $u$ with respect to $\eta$, if

$$
u+\operatorname{t\eta }(u, v) \in K, \quad \forall(v, t) \in K \times[0,1] .
$$

The set $K$ is called an invex set with respect to $\eta$, if $K$ is invex at each $u \in K$. From now on, $K$ denotes a nonempty invex set in $X$ with respect to $\eta$.

Definition 2. The function $f: K \rightarrow \mathbb{R}$ is called preinvex with respect to $\eta$ if, for each $u, v \in K$ and each $t \in[0,1]$, we have

$$
f(u+t \eta(u, v)) \leq(1-t) f(u)+t f(v) .
$$

Let $f: K \times K \rightarrow \mathbb{R}$ be a real-valued bifunction. The invex equilibrium (equilibrium-like) problem (for short, IEP) is to find $u \in K$ such that for all $v \in K$

$$
f(u, v) \geq 0 .
$$

It is well known that the IEP is closely related to the problem of finding $u \in K$ such that

$$
f(v, u) \leq 0, \quad \forall v \in K,
$$

which is called Minty equilibrium-like problem (MIEP) (note that the problem was called dual equilibrium in $[7,10]$ when $K$ is a convex set).

We designate by $S(f, K)$ the solution set of IEP and by $M(f, K)$ the solution set of MIEP.

If $u \in K$ and there exists an open neighborhood $U$ of $u$ such that

$$
f(v, u) \leq 0, \quad \forall v \in K \cap U,
$$

then $u$ is a solution of the local Minty equilibrium-like problem and the solution set of it will be denoted by $M_{L}(f, K)$.

Definition 3. We say that bifunction $f: K \times K \rightarrow \mathbb{R}$ is $\eta$-upper sign continuous, if for any $u, v \in K$, the following implication holds:

$$
f(u+t \eta(u, v), v) \geq 0, \quad \forall t \in] 0,1[\Longrightarrow f(u, v) \geq 0 .
$$

Remark that if, for all $u, v \in K$, we set $\eta(u, v)=v-u$, then the definition reduces to the upper sign continuity introduced by Bianchi and Pini [7]. It is worth noting that the idea of upper sign continuity was first introduced by Hadjisavvas [8]. In what follows we extend the upper sign continuity given [8] for a set-valued mapping whose domain is not necessarily convex.

The following definition extends the definition of the upper sign continuity introduced in [8] for set-valued mappings whose domains are not necessarily convex.
Definition 4. A set-valued map $T: K \rightarrow 2^{X^{*}}$ is said to be $\eta$ upper sign continuous, if for any $u, v \in K$ and for all $t \in] 0,1[$ we have

$$
\inf _{u_{t}^{*} \in T\left(u_{t}\right)}\left\langle u_{t}^{*}, \eta(u, v)\right\rangle \geq 0 \Longrightarrow \sup _{u^{*} \in T(u)}\left\langle u^{*}, \eta(u, v)\right\rangle \geq 0,
$$

where $u_{t}=u+\operatorname{t\eta }(u, v)$.

Note that if the set-valued mapping $T: K \rightarrow 2^{X^{*}}$ has $w^{*}$-compact values and we define $f_{T}: K \times K \rightarrow \mathbb{R}$ by

$$
f_{T}(u, v)=\inf _{u^{*} \in T(u)}\left\langle u^{*}, \eta(u, v)\right\rangle
$$

then we cannot deduce $\eta$-upper sign continuity of $f_{T}$ by $T$ and vice versa. Hence a natural question is how the definition of $\eta$-upper sign continuity can be modified such that the former notion of the upper sign continuity implies the other one. The first time Castellani and Giuli in [9] answered the question by proposing a new definition for mappings whose domains are convex. In the next definition we extend their definition for mappings whose domains are invex.

Definition 5. We say that the bifunction $f: K \times K \rightarrow \mathbb{R}$ has $\eta$-upper sign property (with respect to the first variable) at $u \in K$ if there exists an open neighborhood $U$ of $u$ such that, for any $v \in K \cap U$, the following implication holds:

$$
\left(f\left(u_{t}, u\right) \leq 0 \quad \forall t \in\right] 0,1[) \Longrightarrow f(u, v) \geq 0,
$$

where $u_{t}=u+t \eta(u, v)$.

The following result establishes sufficient conditions under which a mapping has upper sign property.

Lemma 6 (see [9, Lemma 3]). Let $K$ be a convex set and $f$ an equilibrium bifunction (i.e., $f: K \times K \rightarrow \mathbb{R}$ with $f(x, x)=0$, for all $x \in K)$ such that for all $u, v_{1}, v_{2} \in K$ it holds that

$$
\begin{aligned}
& f\left(u, v_{1}\right) \leq 0, \\
& f\left(u, v_{2}\right)<0 \Longrightarrow f\left(x, z_{t}=(1-t) v_{1}+t v_{2}\right) \leq 0, \quad \forall t \in(0,1) .
\end{aligned}
$$

If $f$ is upper sign continuous then it has the upper sign property.

It is a natural question: is the result of the above lemma still valid when the convexity on $K$ and the upper sign continuity have been relaxed? The next result provides an affirmative answer.

Proposition 7. Let $X$ be a topological vector space and $K$ an invex subset of $X$ with respect to $\eta$. Assume that the bifunction $f: K \times K \rightarrow \mathbb{R}$ satisfies the following conditions:

(i) $f(u, u)=0$ for every $u \in K$,

(ii) If $f(u, v)<0$, then $f\left(u, u_{t}\right)<0$, for all $\left.t \in\right] 0,1[$, where $u_{t}=u+t \eta(u, v)$.

(iii) There exists $\left.t_{0} \in\right] 0,1\left[\right.$ such that $0 \leq f\left(u, u_{t_{0}}\right)+$ $f\left(u_{t_{0}}, u\right)$, for every $u \in K$ where $u_{t_{0}}=u+t_{0} \eta(u, v)$.

Then $f$ has $\eta$-upper sign property. 
Proof. On the contrary, suppose that there exist $u, v \in K$ such that $f\left(u_{t}, u\right) \leq 0$, for all $\left.t \in\right] 0,1[$, and $f(u, v)<0$. By (ii) $f\left(u, u_{t}\right)<0$ for all $\left.t \in\right] 0,1$ [. Now it follows from (iii) that there exists $\left.t_{0} \in\right] 0,1$ [ such that

$$
0 \leq f\left(u, u_{t_{0}}\right)+f\left(u_{t_{0}}, u\right)<0,
$$

which is a contradiction. This completes the proof.

The following example satisfies all the assumptions of Proposition 7 while it cannot fulfill all the assumptions of Lemma 6.

Example 8. Let $K=[0,1]$ and $f: K \times K \rightarrow \mathbb{R}$ such that

$$
f(x, y)= \begin{cases}y-1, & x=1,0 \leq y \leq 1 \\ |x-y|, & 1 \geq y \geq x \geq 0 \\ 1, & 1>x>y>0\end{cases}
$$

It is straightforward to check that $f$ satisfies conditions of Proposition 7 and so it has upper sign property. But it is not upper sign continuous, because if we take $x=1$ and $y=0$, then we have

$$
f\left(x_{t}=t x+(1-t) 0,0\right)=f(t, 0)=1 \geq 0, \quad \forall t \in(0,1),
$$

whereas $f(1,0)=-1<0$. Hence the hypothesis of Lemma 6 cannot work.

The next example shows that condition (iii) of Proposition 7 is essential.

Example 9. let $K=[0,1]$ and $f: K \times K \rightarrow \mathbb{R}$ as

$$
f(x, y)=-|x-y| \text {. }
$$

Then $f$ satisfies (i) and (ii) of Proposition 7 but not (iii). Note that $f$ is upper sign continuous but does not have upper sign property.

It is worth noting that Example 9 indicates that upper sign continuity cannot imply condition (iii) of Proposition 7. Now one may ask the converse. The following example answers the question.

Example 10. Let $f: \mathbb{R} \times \mathbb{R} \rightarrow \mathbb{R}$ be defined by

$$
f(x, y)= \begin{cases}0, & x=y, \\ 1, & x \in Q \text { (the rational numbers), } \\ -1, & x \in Q^{c} \text { (the irrational numbers). }\end{cases}
$$

It is easy to verify that $f$ satisfies condition (iii) while $f$ is not upper sign continuous.

\section{Application of the $\eta$-Upper Sign Property}

In this section we establish a link between the solution set of the equilibrium-like problem (that is, $S(f, K)$ ) and the solution set of the local Minty equilibrium-like problem $\left(M_{L}(f, K)\right)$. The result presented is another version of Theorem 1 of [9] without assuming convexity on the set and upper sign continuity on the map.
Theorem 11. Let $f: K \times K \rightarrow \mathbb{R}$ be a bifunction with $\eta$-upper sign property and

$$
\eta(u, u+t \eta(u, v))=t \eta(u, v) \quad \forall u, v \in K, \forall t \in[0,1] .
$$

If $f$ satisfies the following condition:

$$
\left.f(u, v)<0 \Longrightarrow f\left(u, u_{t}=u+t \eta(u, v)\right)<0, \quad \forall t \in\right] 0,1[
$$

then $M_{L}(f, K) \subseteq S(f, K)$.

Proof. Let $u \in M_{L}(f, K)$ and $v \in K$ be arbitrary. Therefore by definition of $M_{L}(f, K)$ there exists an open neighborhood $U$ of $u$ such that

$$
f(w, u) \leq 0 \quad \forall w \in K \cap U .
$$

Since $U-u$ is a neighbourhood of 0 , there exists $\left.t_{0} \in\right] 0,1[$ such that $t \eta(u, v) \in U-u$ for all $t \in\left[0, t_{0}\right]$. Let $\bar{v}=u+$ $t_{0} \eta(u, v)$ and $v_{t}=u+t \eta(u, \bar{v})$ for $t \in[0,1]$. Then $v_{t} \in U \cap$ $K$, since $v_{t}=u+t \eta\left(u, u+t_{0} \eta(u, v)\right)=u+t t_{0} \eta(u, v)$ and $t t_{0} \eta(u, v) \in U-u$. Hence, by our assumption on $u, f\left(v_{t}, u\right) \leq$ 0 . Now from $\eta$-upper sign property of $f$, we have $f(u, \bar{v}) \geq$ 0 . So from (17) we conclude $f(u, v) \geq 0$ and this completes the proof.

Remark 12. There are many functions $\eta: K \times K \rightarrow X$ which satisfy $\eta(u, u+t \eta(u, v))=t \eta(u, v)$. For instance, let $K=\mathbb{R}$

$$
\eta(u, v)=\left\{\begin{array}{l}
v-u, \quad v \geq 0, u \geq 0 \\
v-u, \quad v \leq 0, u \leq 0 \\
u, \quad \text { otherwise }
\end{array}\right.
$$

It is clear that $K$ is an invex set and $\eta$ satisfies (17).

If $\eta(u, v)=v-u$, then $K$ will be a convex set and clearly the condition (17), that is,

$$
\eta(u, u+t \eta(u, v))=\operatorname{t\eta }(u, v), \quad \forall u, v \in K, \forall t \in[0,1],
$$

is automatically satisfied.

Corollary 13. If the bifunction $f$ satisfies all the assumptions of Proposition 7 and

$$
\eta(u, u+t \eta(u, v))=t \eta(u, v) \quad \forall u, v \in K, \forall t \in[0,1],
$$

then $M_{L}(f, K) \subseteq S(f, K)$. This means that the upper sign continuity property in Theorem 11 can be substituted by the conditions of Proposition 7.

Remark 14. One can see that Theorem 11 and Corollary 13 extend the corresponding result given in [9] for bifunctions whose domains are not necessarily convex with weaker hypothesis. To verify this one can consider Example 8 which satisfies all the assumptions of Theorem 11 as well as Corollary 13 and $M_{L}(f, K)=\{\} \subseteq S(f, K)=[0,1)$ while $f$ is not upper sign continuous. Hence, even when $K$ is a convex set, our conditions are weaker than the conditions of Lemma 3 in [9] and consequently Lemma 2.1 in [10] and Lemma 2.1 in [7]. 
Proposition 15. Assume that $f$ satisfies condition (ii) of Proposition 7 and, for all $u, v \in X$, we have $M(f,[u, v]) \subseteq$ $S(f,[u, v])$ where $[u, v]=u+t \eta(u, v), t \in[0,1]$. Then $f$ has $\eta$-upper sign property.

Proof. Assume that $f$ does not have $\eta$-upper sign property at $u \in U$; thus, for all open neighborhoods $U$ of $u$, there exists $v \in U \cap K$ such that $f\left(u_{t}, u\right) \leq 0$ for all $\left.t \in\right] 0,1[$, but $f(u, v)<0$. From condition (ii) of Proposition 7, we have $f\left(u, u_{t}\right)<0$ for all $\left.t \in\right] 0,1\left[\right.$, where $u_{t}=u+t \eta(u, v)$. Clearly $u \in M\left(f,\left[u, u_{t}\right]\right)$; therefore $u \in S\left(f,\left[u, u_{t}\right]\right)$, which implies that $f\left(u, u_{t}\right) \geq 0$ and a contradiction occurs.

\section{Relations between Solution Set of Variational-Like Inequalities and Equilibrium-Like Problems}

In this section we investigate the relationship between $\eta$ upper sign property and upper sign continuity in the setting of variational inequality. Also we are going to establish a link between the solution sets of variational inequality and Minty variational inequality.

Variational-like inequality problem is formulated as follows:

find $u \in K$ such that $\exists u^{*} \in T(u)$ with $\left\langle u^{*}, \eta(u, v)\right\rangle \geq 0$

$$
\forall v \in K \text {. }
$$

And the Minty variational-like inequality problem is formulated as follows:

find $u \in K$ such that $\left\langle v^{*}, \eta(u, v)\right\rangle \geq 0 \quad \forall v \in K, v^{*} \in T(v)$.

We denote by $S(T, K)$ and $M(T, K)$ the solution sets of the variational inequality problem and the Minty variational inequality problem, respectively.

The set-valued map $T: K \rightarrow 2^{X^{*}}$ is said to be $\eta$ pseudomonotone on $K$ if, for all $u, v \in K, \exists u^{*} \in T(u)$ such that

$$
\left\langle u^{*}, \eta(u, v)\right\rangle \geq 0 \Longrightarrow\left\langle v^{*}, \eta(u, v)\right\rangle \geq 0 \quad \forall v^{*} \in T(v) .
$$

Proposition 16. Let $T: K \rightarrow 2^{X^{*}}$ be a set-valued map with $w^{*}$-compact value and $f_{T}$ be defined as follows:

$$
f_{T}(u, v)=\sup _{u^{*} \in T(u)}\left\langle u^{*}, \eta(u, v)\right\rangle .
$$

If $\eta(\cdot, \cdot): K \times K \rightarrow X$ satisfies

$$
-\eta\left(u_{t}, u\right)=\operatorname{t\eta }(u, v) \quad \forall t \in[0,1],
$$

then $T$ is $\eta$-upper sign continuous if and only if $f_{T}$ has $\eta$-upper sign property.

Proof. Let $T$ be $\eta$-upper sign continuous and take $u, v \in K$ such that

$$
\left.f_{T}\left(u_{t}=u+t \eta(u, v), u\right) \leq 0 \quad \forall t \in\right] 0,1[.
$$

So

$$
f_{T}\left(u_{t}, u\right)=\sup _{u^{*} \in T(u)}\left\langle u_{t}^{*}, \eta\left(u_{t}, u\right)\right\rangle \leq 0,
$$

and so it follows from the property of the least upper bound that

$$
\inf _{u^{*} \in T(u)}\left\langle u_{t}^{*},-\eta\left(u_{t}, u\right)\right\rangle \geq 0
$$

and, by using the hypothesis on $\eta$, we get

$$
\inf _{u^{*} \in T(u)}\left\langle u_{t}^{*}, t \eta(u, v)\right\rangle \geq 0
$$

and since $T$ is $\eta$-upper sign continuity we have

$$
f_{T}=\sup _{u^{*} \in T(u)}\left\langle u^{*}, \eta(u, v)\right\rangle \geq 0 .
$$

Therefore $f_{T}$ has $\eta$-upper property.

Conversely, let $f_{T}$ have $\eta$-upper sign property. We want to show that $T$ is $\eta$-upper sign continuous. To see this, assume $u, v \in K$ such that, for all $t \in] 0,1[$,

$$
\inf _{u^{*} \in T(u)}\left\langle u_{t}^{*}, \operatorname{t\eta }(u, v)\right\rangle \geq 0
$$

where $u_{t}=u+t \eta(u, v)$. So it follows from $-\eta\left(u_{t}, u\right)=t \eta(u, v)$ that

$$
\left.\inf _{u^{*} \in T(u)}\left\langle u_{t}^{*},-\eta\left(u_{t}, u\right)\right\rangle \geq 0, \quad \forall t \in\right] 0,1[
$$

Hence

$$
\begin{aligned}
f_{T}\left(u_{t}, u\right) & =\sup _{u_{t}^{*} \in T\left(u_{t}\right)}\left\langle u_{t}^{*}, \eta\left(u_{t}, u\right)\right\rangle \\
& \left.=-\inf _{u_{t}^{*} \in T\left(u_{t}\right)}\left\langle u_{t}^{*},-\eta\left(u_{t}, u\right)\right\rangle \leq 0, \quad \forall t \in\right] 0,1[,
\end{aligned}
$$

and we can deduce from the $\eta$-upper sign property of $f_{T}$ that

$$
\sup _{u^{*} \in T(u)}\left\langle u^{*}, \eta(u, v)\right\rangle=f_{T}(u, v) \geq 0,
$$

and this completes the proof.

Remark that if we assume that $K$ is convex and take $\eta(u, v)=v-u$; then it is clear that

$$
-\eta\left(u_{t}, u\right)=t \eta(u, v), \quad \forall t \in[0,1] .
$$

This means that in the setting of convexity Proposition 16 collapses to the corresponding result given in [9] and moreover there are many examples of $\eta$ which are not equal to the usual case $\eta(u, v)=v-u$. For instance, see the following example.

Example 17. Let $K=\mathbb{R} \backslash\{0\}$ and

$$
\eta(u, v)= \begin{cases}v-u, & v \geq 0, u \geq 0 \\ v-u, & v \leq 0, u \leq 0 \\ -u, & \text { otherwise }\end{cases}
$$

It is clear that $K$ is an invex set (in here not convex) and that $\eta$ satisfies the condition $-\eta\left(u_{t}, u\right)=t \eta(u, v)$. 
The next result provides a sufficient condition under which the set-valued map $T$ is $\eta$-upper sign continuous.

Corollary 18. Let $T: X \rightarrow 2^{X^{*}}$ be a set-valued map with $w^{*}$ compact values and $K$ an invex subset of $X$ with $-\eta\left(u_{t}, u\right)=$ $\operatorname{t\eta }(u, v)$. If $M(T,[u, v]) \subseteq S(T,[u, v])$ for all $u, v \in K$; then $T$ is $\eta$-upper sign continuous on $K \times K$.

Corollary 19. Under conditions of previous corollary, $T$ is $\eta$ pseudomonotone and $\eta$-upper sign continuous on $K$ if and only if $M(T, K)=S(T, K)$.

\section{Existence Results}

Let $(X, d)$ be a complete metrizable topological vector space, let $K$ be a nonempty closed invex subset of $X$, and let $f: K \times$ $K \rightarrow \mathbb{R}$ be a bifunction. For a given $\varepsilon>0$, we consider the following sets:

$$
\begin{aligned}
& A(\varepsilon)=\{u \in K: F(u, v) \geq-\varepsilon d(u, v), \forall v \in K\}, \\
& B(\varepsilon)=\{u \in K: F(v, u) \leq-\varepsilon d(u, v), \forall v \in K\} .
\end{aligned}
$$

Definition 20. The equilibrium-like problem (IEP) is called well posed if, for every $\varepsilon>0$, we have

$$
\begin{gathered}
A(\varepsilon) \neq 0, \\
D(A(\varepsilon)) \longrightarrow 0, \quad \text { as } \varepsilon \longrightarrow 0,
\end{gathered}
$$

where $D(A(\varepsilon))$ denotes the diameter of the set $A(\varepsilon)$.

It is clear that the solution set of (IEP), that is, $S(f, K)$, equals the set $\bigcap_{\varepsilon>0} A(\varepsilon)$.

Theorem 21. If the bifunction $(u, v) \rightarrow f(u, v)+\varepsilon d(v, u)$ is pseudomonotone and satisfies all the assumptions of Proposition 7 then $A(\varepsilon)=B(\varepsilon)$.

Proof. If $f$ is pseudomonotone, then $S(f, K) \subseteq M(f, K)$. Hence, in Proposition 7, if $f$ is also pseudomonotone, then we have $M(f, K)=S(f, K)$. So the assertion follows.

Since Proposition 7 is stronger than the Lemma 1.2 in [11], the following theorem provides a stronger result than Theorem 2.2 in [11].

Theorem 22. If $A(\varepsilon)$ is closed for all $\varepsilon>0$ and the IEP is well posed, then the equilibrium-like problem has a unique solution. In addition if the bifunction $(u, v) \mapsto f(u, v)+\varepsilon d(v, u)$ is pseudomonotone, then the solution set MIEP is a singleton and equals the solution set IEP.

Now we recall a so-called theorem by Fan [12] that is a fundamental result in the framework of equilibrium problem and variational inequality.

Theorem 23. Let $X$ be a real Hausdorff topological vector space, $K$ a subset of $X$, and $T: K \rightarrow 2^{X^{*}}$ a set-valued map satisfying the following conditions: (a) Tis a KKM-map, that is, for any $n \in \mathbb{N}$ and $u_{1}, \ldots, u_{n} \in$ $K$,

$$
\operatorname{conv}\left\{u_{1}, \ldots, u_{n}\right\} \subseteq \bigcup_{i=1}^{n} T\left(u_{i}\right)
$$

(b) $T(x)$ is closed for each $x \in K$.

(c) There exists $u \in K$ such that $T(u)$ is compact. Then

$$
\bigcap_{u \in K} T(u) \neq \emptyset
$$

Define the map $F: K \rightarrow 2^{X}$ for every $u \in K$ as follows:

$$
F(u)=\{u \in K: f(u, v) \leq 0\} .
$$

Theorem 24. Let $K$ be weakly compact and F a KKM setvalued map such that $F(u)$ is weakly closed for every $u \in K$. Then $M_{L}(f, K)$ is nonempty.

Proof. Since $F$ is a KKM map, so Theorem 23 implies that $\bigcap_{u \in K} F(u) \neq \emptyset$ and by definition of $F(u)$ we have $\bigcap_{u \in K} F(u) \subseteq M_{L}(f, K)$.

Corollary 25. Supposing that conditions of Proposition 7 and Theorem 24 are satisfied, then $S(f, K) \neq \emptyset$.

\section{Conflict of Interests}

The authors declare that there is no conflict of interests regarding the publication of this paper.

\section{Acknowledgment}

This research was supported in part by National Science Council of the Republic of China.

\section{References}

[1] M. A. Hanson, "On sufficiency of the Kuhn-Tucker conditions," Journal of Mathematical Analysis and Applications, vol. 80, no. 2, pp. 545-550, 1981.

[2] E. Blum and W. Oettli, "From optimization and variational inequalities to equilibrium problems," The Mathematics Student, vol. 63, no. 1-4, pp. 123-145, 1994.

[3] M. A. Noor and W. Oettli, "On general nonlinear complementarity problems and quasi-equilibria," Le Matematiche, vol. 49, no. 2, pp. 313-331, 1994.

[4] Giannessi, F. Maugeri A, and P. M. Pardalos, Equilibrium Problems: Nonsmooth Optimization and Variational Inequality Models, Kluwer Academic, Dordrecht, The Netherlands, 2001.

[5] M. A. Noor, "Auxiliary principle technique for equilibrium problems," Journal of Optimization Theory and Applications, vol. 122, no. 2, pp. 371-386, 2004.

[6] M. A. Noor, "Invex equilibrium problems," Journal of Mathematical Analysis and Applications, vol. 30, pp. 463-475, 2005.

[7] M. Bianchi and R. Pini, "Coercivity conditions for equilibrium problems," Journal of Optimization Theory and Applications, vol. 124, no. 1, pp. 79-92, 2005. 
[8] N. Hadjisavvas, "Continuity and maximality properties of pseudomonotone operators," Journal of Convex Analysis, vol. 10, pp. 459-469, 2003.

[9] M. Castellani and M. Giuli, "Refinements of existence results for relaxed quasimonotone equilibrium problems," Journal of Global Optimization, vol. 57, no. 4, pp. 1213-1227, 2013.

[10] A. P. Farajzadeh and J. Zafarani, "Equilibrium problems and variational inequalities in topological vector spaces," Optimization, vol. 59, no. 3-4, pp. 485-499, 2010.

[11] A. P. Farajzadeh and M. A. Noor, "On dual invex Ky Fan inequalities," Journal of Optimization Theory and Applications, vol. 145, no. 2, pp. 407-413, 2010.

[12] K. Fan, "A minimax inequality and applications," in Inequalities III, O. Shisha, Ed., pp. 103-113, Academic Press, New York, NY, USA, 1972. 


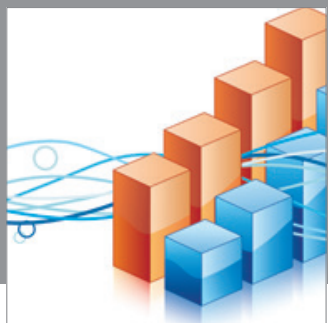

Advances in

Operations Research

mansans

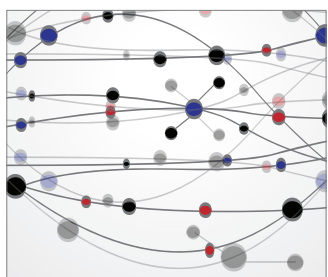

The Scientific World Journal
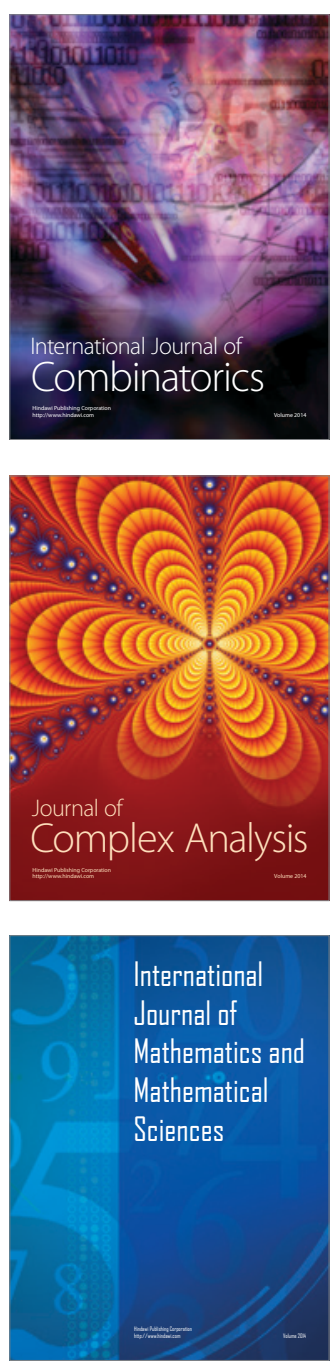
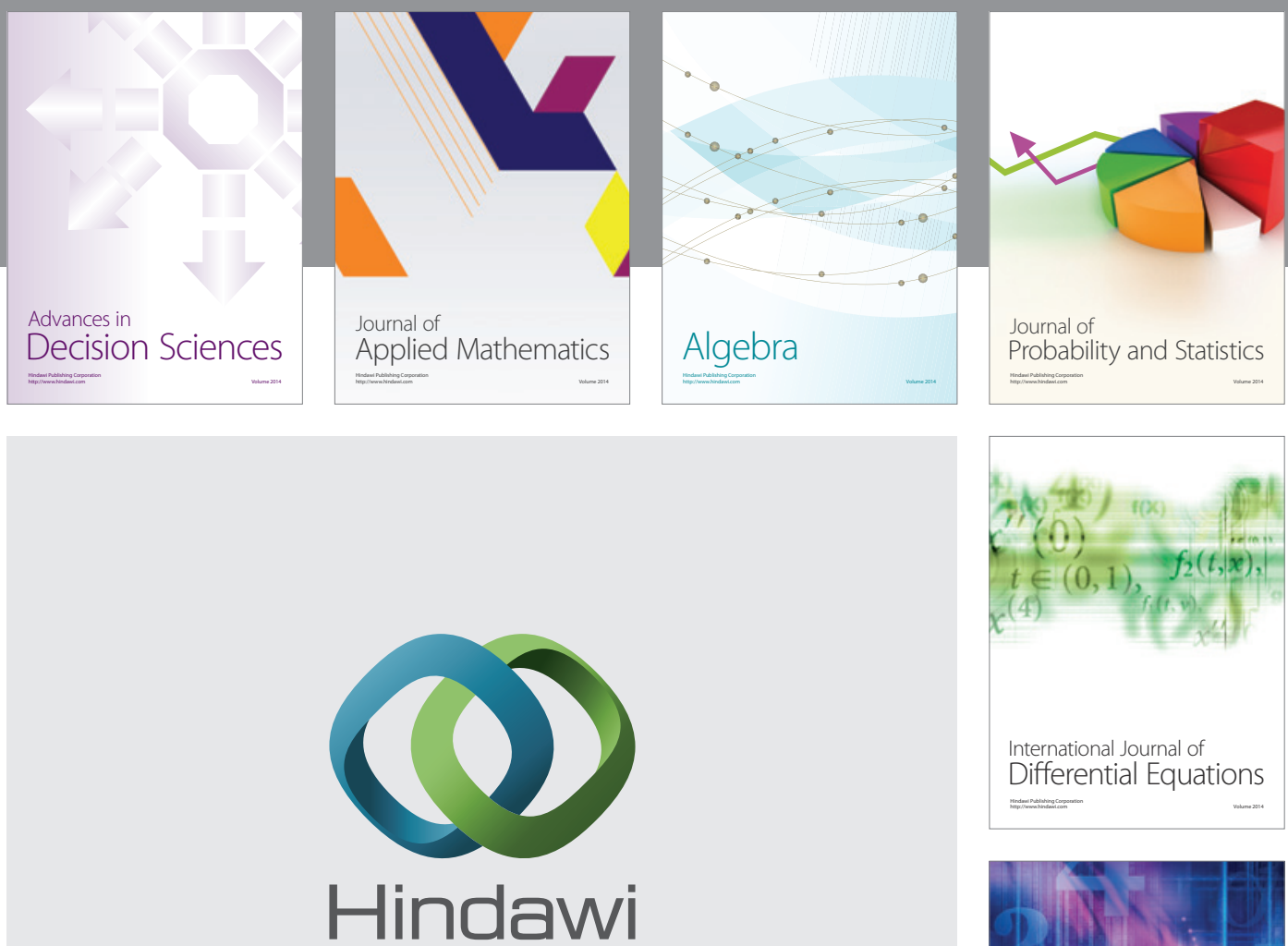

Submit your manuscripts at http://www.hindawi.com
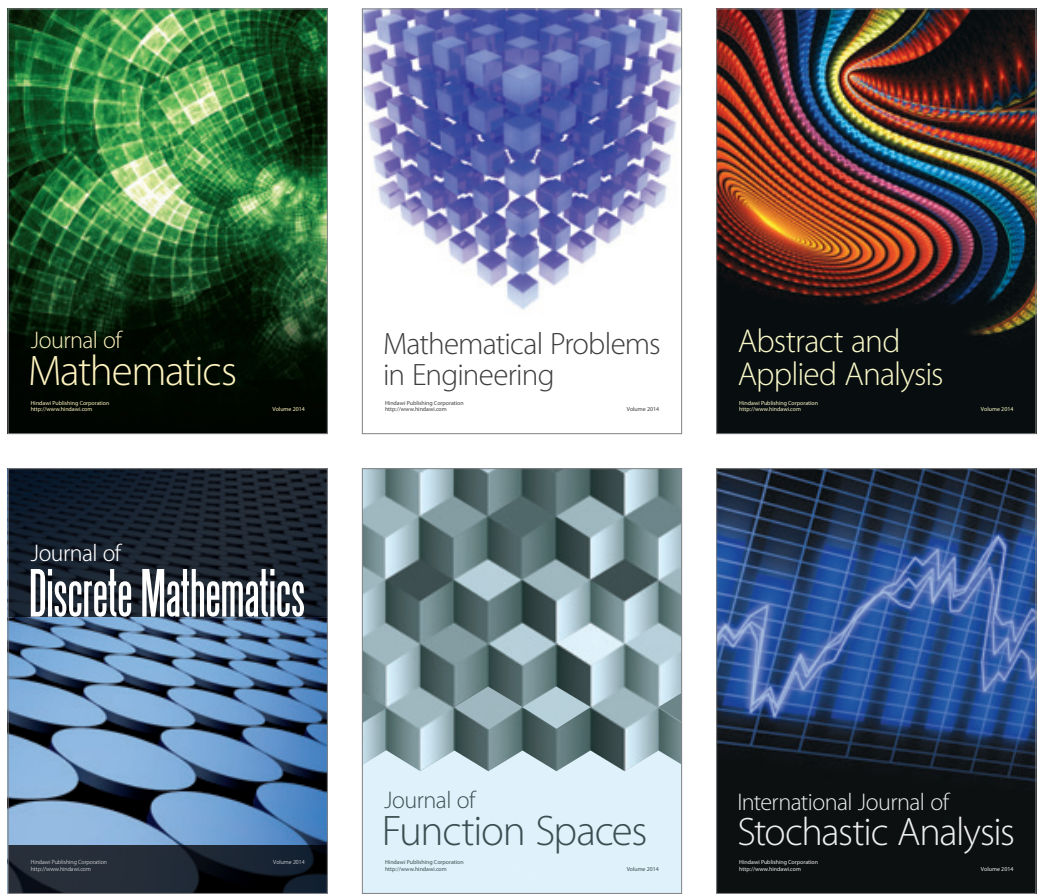

Journal of

Function Spaces

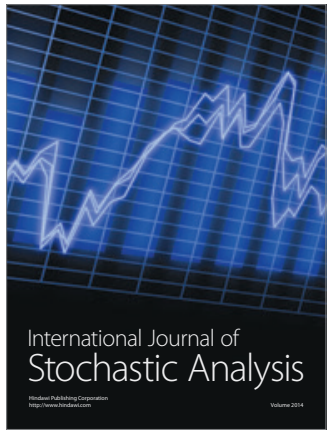

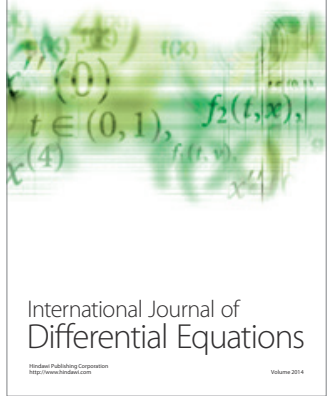
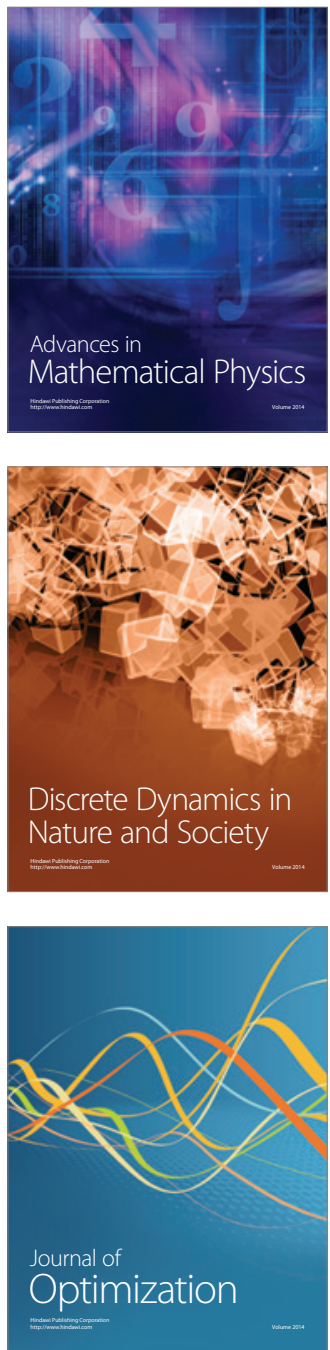